\title{
Angioplastia Pulmonar com Balão Percutâneo Sistemática com Estadiamento em Hipertensão Pulmonar Tromboembólica Crônica Inoperável Grave
}

\author{
Systematic Staged Percutaneous Balloon Pulmonary Angioplasty in Severe Inoperable Chronic \\ Thromboembolic Pulmonary Hypertension
}

Kazuhiro Dan, ${ }^{10}$ Akira Shionoda, ${ }^{1}$ Hiromi Matsubara ${ }^{2}$

Ichinomiya Nishi Hospital,'1 Ichinomiya - Japão

Okayama Medical Center, ${ }^{2}$ Okayama - Japão

\section{Introdução}

O tratamento para hipertensão pulmonar tromboembólica crônica (HPTEC) é limitado a endarterectomia pulmonar (PEA), terapia medicamentosa e angioplastia pulmonar percutânea com balão (APB). ${ }^{1} \mathrm{O}$ tratamento padrão-ouro é a PEA, e pacientes com HPTEC e lesões proximais são geralmente bons candidatos cirúrgicos. Complicações perioperatórias e hipertensão pulmonar persistente devido à endarterectomia incompleta ou vasculopatia secundária são problemas típicos após o procedimento. ${ }^{2}$ Uma metanálise mostrou que a eficácia da terapia medicamentosa para HPTEP grave é limitada, e muitos pacientes não chegam ter a artéria pulmonar (AP) suficientemente reduzida, mesmo havendo uma melhora marginal da tolerância ao exercício. ${ }^{3}$ A APB percutânea foi reportada pela primeira vez em 2001, mas sua segurança não foi comprovada na época. ${ }^{4}$ Recentemente, há apenas uma década, a hipertensão pulmonar foi associada pela primeira vez a um prognóstico ruim. Felizmente, os tratamentos melhoraram drasticamente desde então, particularmente para pacientes com HPTEC. A APB ainda é uma estratégia desafiadora, pois tem a limitação de um operador especializado e instalação específica, mas seus resultados têm melhorado. ${ }^{5}$

\section{Relato de Caso}

Paciente do sexo feminino, 76 anos, 1,45 m de altura, $40 \mathrm{~kg}$, apresentou-se ao serviço com história de dispneia ao esforço havia três meses. Sem histórico de trombose venosa profunda ou embolia pulmonar aguda. Uma semana antes da internação, a dispneia piorou (classe IV da New York Heart Association), a paciente desenvolveu edema nas pernas e tornou-se incapaz de andar. $\mathrm{Na}$ admissão, tinha pressão arterial de 210/95 mmHg, frequência cardíaca de 85 bpm,

\section{Palavras-chave}

Angioplastia Pulmonar com Balão, Hipertensão Pulmonar, Embolia Pulmonar, Fragilidade, Idosos.

Correspondência: Kazuhiro Dan •

Ichinomiya Nishi Hospital - Kaimei-hira 1, Ichinomiya, Aichi 494-0001, Ichinomiya 4940001- Japão

E-mail: dan27k@gmail.com

Artigo recebido em 17/10/2019, revisado em 27/01/2020, aceito em $16 / 03 / 2020$

DOI: https://doi.org/10.36660/abc.20190717

$\mathrm{SpO}_{2}$ de $80 \%$ (ar ambiente) e frequência respiratória de 32 respirações por minuto. O eletrocardiograma revelou ritmo sinusal e hipertrofia ventricular direita (HVD) (Figura 1A). Os exames laboratoriais mostraram creatinina $0,84 \mathrm{mg} /$ dl, hemoglobina $17,4 \mathrm{~g} / \mathrm{dl}$ e peptídeo natriurético cerebral (BNP) 1000 pg/ml. Nenhuma evidência de doença vascular do colágeno foi encontrada. A ecocardiografia transtorácica revelou insuficiência cardíaca direita [dilatação do átrio e ventrículo direitos; pressão sistólica da AP estimada em 58 $\mathrm{mmHg} ; 6 \%$ de alteração da área fracionária do ventrículo direito; derrame pericárdico] com contração ventricular esquerda preservada (fração de ejeção: 65\%) (Figura 1B).

Foi submetida a um cateterismo cardíaco direito, que revelou pressão da AP a 60/38 (47) $\mathrm{mmHg}$, pressão de oclusão da artéria pulmonar expiratória final (PAWP) de $6 \mathrm{mmHg}$, índice cardíaco de 2,17 L/min e índice de resistência vascular pulmonar de 18,89 unidades Wood $\bullet \mathrm{m}^{2}$. A angiografia coronária mostrou-se normal. Uma tomografia computadorizada de tórax com contraste não mostrou evidências de embolia pulmonar aguda (Figura 1D-F). Angiografia da AP revelou lesões em teias e de oclusão total e subtotal em artérias segmentares a subsegmentares bilaterais (Figura 2A). Uma cintilografia de perfusão pulmonar mostrou múltiplas lesões bilaterais (Figura 3A).

A paciente recebeu infusões contínuas de heparina e dobutamina em baixas doses durante um mês; no entanto, sua condição não melhorou e ela foi diagnosticada com HPTEC. Até três meses antes da internação, realizava todas as atividades de forma independente, porém, por ser idosa e frágil, referiu estar completamente acamada por uma semana antes da internação. Sua fragilidade (escala clínica canadense 8) e múltiplas lesões distais fizeram dela uma candidata não favorável à cirurgia, então a equipe médica decidiu, após discussões com um cirurgião cardíaco, realizar APB por se tratar de um procedimento menos invasivo e de menor risco.

Os vasos-alvo foram as artérias pulmonares direitas (A1, A2, A3, A5, A7 e A8) e esquerdas (A 3, A4, A6 e A10). $\mathrm{O}$ procedimento foi realizado com um sistema de cateterguia de 0,014 polegadas, semelhante a uma intervenção coronária percutânea. Um cateter-guia 6 French Amplatz esquerdo foi inserido no sentido de um ramo da AP através da veia femoral direita. Foi realizada com dois tipos de cateter-guia de baixo peso (B-pahm 0,6g, Japan Lifeline, Tóquio, Japão) (Chevalier Floppy 2g, FMD, Tóquio, Japão), com suporte de um cateter balão (CB). No primeiro procedimento, a APB foi iniciada pela parte anterior (A3 direita e A5 A3 esquerda) usando um CB semicomplacente 


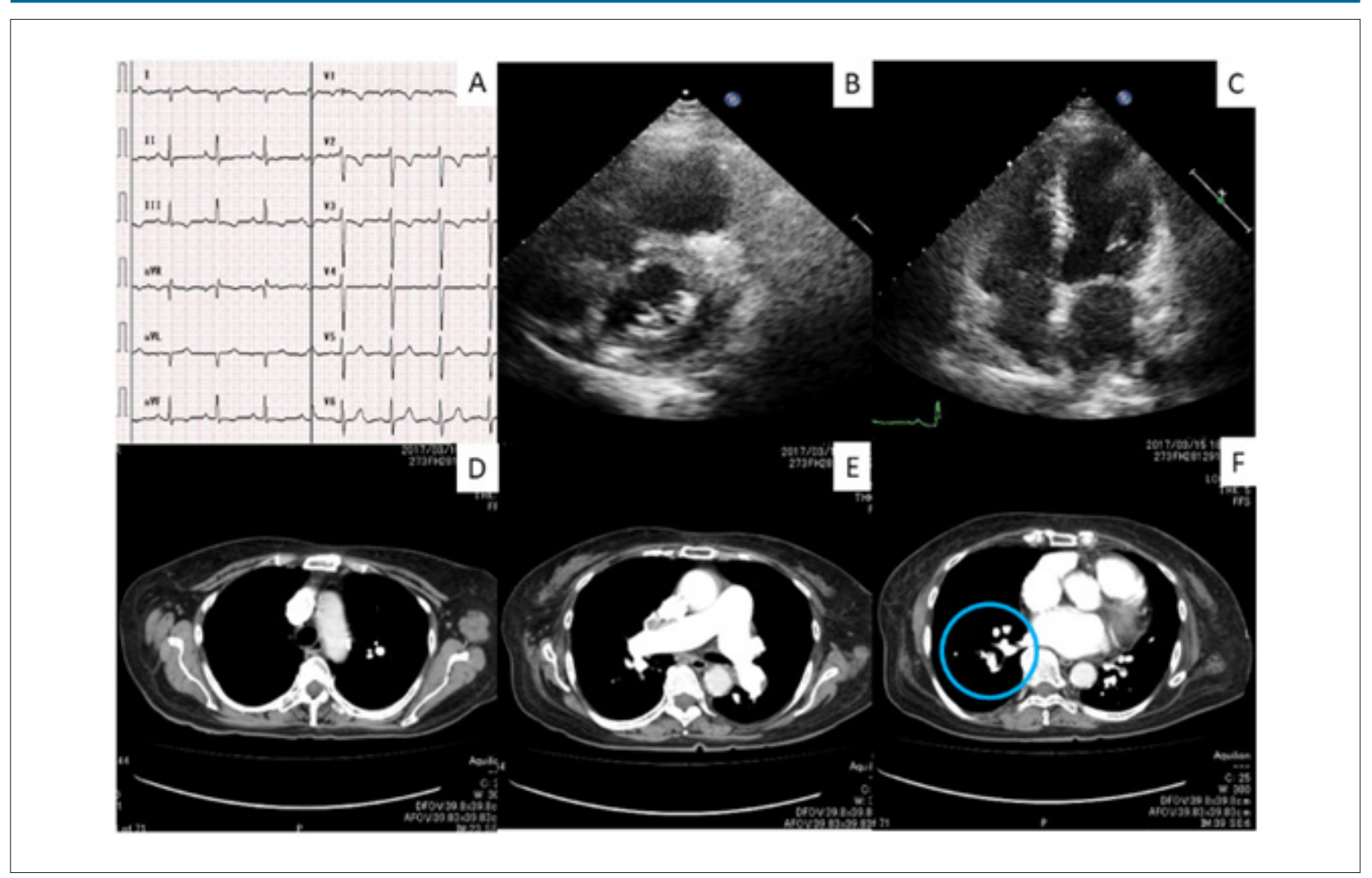

Figura 1 - Exames fisiológicos e radiológicos antes da angioplastia pulmonar com balão. A) Eletrocardiograma na admissão mostrava S-I e T-III. B e C) Ecocardiografia transtorácica (fase diastólica final) mostrando insuficiência cardiaca direita (B) na admissão e insuficiência cardiaca direita normalizada dois anos após a APB (C). D-F) Tomografia computadorizada de tórax com contraste mostrando a área avascular no lobo superior direito (D), sem evidência de embolia pulmonar aguda no tronco da artéria pulmonar (E), e achado semelhante a uma teia no ramo da artéria pulmonar direita 8 (círculo azul em F).

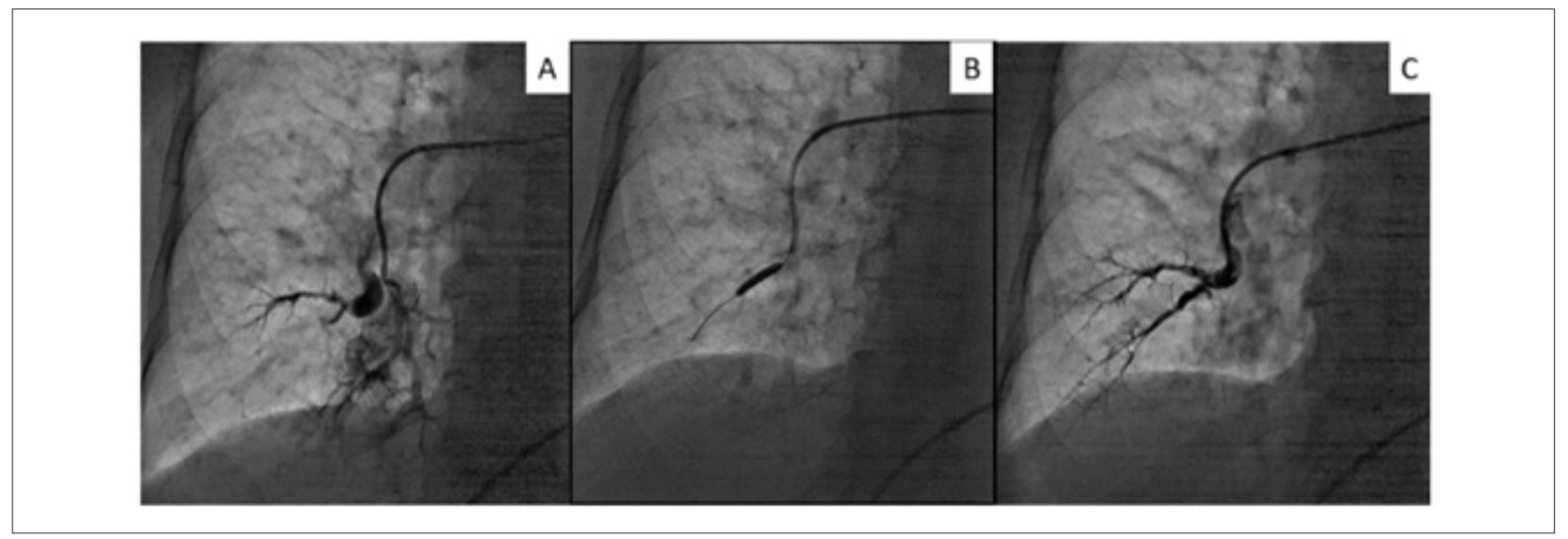

Figura 2 - Angiografia da artéria pulmonar representativa em angioplastia pulmonar com balão sistemática. A: Artéria pulmonar direita ocluída (ramo 8) detectada por angiografia seletiva. B: APB com cateter balão semicomplacente de 3,0 mm. C: Angiografia pulmonar seletiva final após APB sistemática com cateteres balão semicomplacente de 2,0 e $3,0 \mathrm{~mm}$.

de 2,0 mm (Ikazuchi PAD, Kaneka, Osaka, Japão). Auma APB adicional usando $C B$ de $2,0 \mathrm{~mm}$ foi realizada na porção póstero-lateral (A3, A5, A7 e A8 direita, A4, A6 e A10 esquerda) um mês depois. Dois meses mais tarde, expandimos todas as artérias pré-dilatadas com um CB de 3,0 mm (Figura 2B e 2C). A APB foi concluída sem orientação de imagem intravascular e sem complicações, como lesão pulmonar e hemoptise.

Não conseguimos abrir as artérias completamente ocluídas (A 1 e A2 direita); entretanto, a pressão da AP diminuiu para 42/16 (26) $\mathrm{mmHg}$ imediatamente após a APB final, e a pressão da PA média finalmente melhorou para $20 \mathrm{mmHg}$ sem hipoxemia $\left(\mathrm{SpO}_{2}\right.$ 


\section{Relato de Caso}

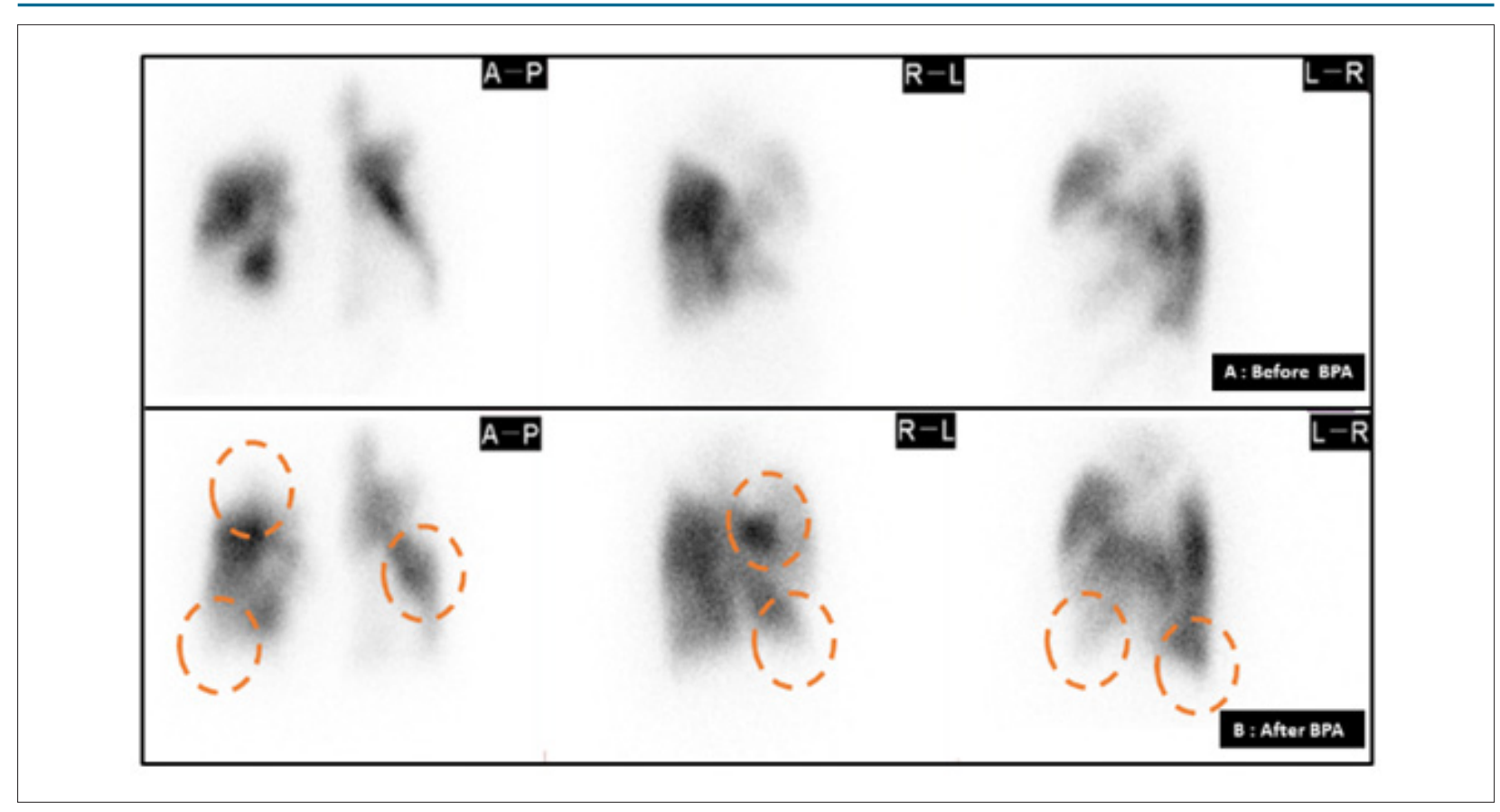

Figura 3 - Cintilografia de perfusão pulmonar antes e depois da angioplastia pulmonar sistemática com balão. $A$ e B) Cintilografia de perfusão pulmonar antes (A) e depois (B) da APB. Os circulos laranja mostram áreas de perfusão melhoradas.

$96 \%$, ar ambiente). Uma nova cintilografia de perfusão pulmonar mostrou melhora da perfusão, com circulação pulmonar adequada em dois terços do leito vascular total da AP (Figura 3B). Ela passou a deambular de forma independente e teve alta hospitalar. Na alta, sua distância de caminhada de seis minutos era de $236 \mathrm{~m}$. Foi prescrito oxigênio suplementar, anticoagulantes e um diurético de baixa dosagem. O BNP diminuiu para 64 $\mathrm{pg} / \mathrm{ml}$. Um ecocardiograma mostrou que as dilatações atrial e ventricular direita haviam se normalizado (Figura 1C). Desde então, a paciente manteve um bom quadro clínico ao longo de dois anos, mas um monitoramento adicional é recomendado.

\section{Discussão}

Nossa paciente apresentou HPTEC grave inoperável, sem trombos na parte principal das artérias pulmonares à tomografia computadorizada. Angiografia pulmonar mostrou artérias pulmonares segmentares ocluídas (A 1, 2, 8 direita e A 10 esquerda) e lesões em teia em outras artérias segmentares ou subsegmentares. ${ }^{6}$

Neste caso, realizamos três sessões separadas de APB; no entanto, sessões mais espaçadas podem ser aceitáveis, dependendo da fragilidade e do estado geral do paciente, a fim de evitar lesão pulmonar (como sangramento) por dano a vaso pulmonar. ${ }^{1,7}$ Também consideramos incompatibilidade ventilação-perfusão e iniciamos o procedimento pela porção anterior para melhorar a hipoxemia. Lesões oclusivas são um preditor de complicações relacionadas à APB. ${ }^{8}$ Em seguida, prosseguimos ao tratamento das artérias obstruídas de forma incompleta, uma vez que as complicações relacionadas ao procedimento podem piorar criticamente a condição hemodinâmica e respiratória da paciente. Foi selecionado um
CB pequeno (de 2,0 mm) para evitar o fluxo sanguíneo de alta pressão e, em seguida, as múltiplas artérias-alvo foram dilatadas.

Após a dilatação por fluxo sanguíneo de entrada por dois meses após a APB inicial, todas as artérias tratadas foram dilatadas com um CB de 3,0 mm, de acordo com o diâmetro anatômico da AP. Um relato recente descreveu que pacientes com HPTEC apresentam aumento na rigidez arterial. ${ }^{9} \mathrm{~A}$ hipertensão arterial sistêmica é incomum na HPTEC, mas, no presente caso, foi normalizada após a APB.

Grupos japoneses relataram resultados melhores em longo prazo associados à APB para pacientes com HPTEC e lesões distais. ${ }^{10}$ Mais estudos observacionais prospectivos e ensaios clínicos randomizados são necessários para comparar a APB e a terapia medicamentosa em pacientes com HPTEC inoperável, determinando assim a eficácia do procedimento.

\section{Conclusão}

A APB sistemática, um tratamento de AP anteroposterior com dois cateteres-balão com diâmetros diferentes (2,0 mm e 3,0 mm), pode ser realizada com segurança, mesmo em pacientes inoperáveis e em condições físicas severas. Hoje em dia, a APB pode não ser o último recurso, mas sim o tratamento de primeira escolha para a população com HPTEC inoperável.

\section{Contribuição dos autores}

Concepção e desenho da pesquisa, Análise e interpretação dos dados e Redação do manuscrito: Dan K; Obtenção de dados: Shionoda A; Revisão crítica do manuscrito quanto ao conteúdo intelectual importante: Matsubara $\mathrm{H}$. 


\section{Potencial conflito de interesses}

Declaro não haver conflito de interesses pertinentes.

\section{Fontes de financiamento}

O presente estudo não teve fontes de financiamento externas.

\section{Referências}

1. Kim NH, Delcroix M, Jais X, Madani MM, Matsubara H, Mayer E, et al. Chronic thromboembolic pulmonary hypertension. Eur Respir J. 2019;53(1):1801915.

2. Mayer E, Jenkins D, Lindner J, D'Armini A, Kloek J, Meyns B, et al. Surgical management and outcome of patients with chronic thromboembolic pulmonary hypertension: results from an international prospective registry. J Thorac Cardiovasc Surg. 2011;141(3):702-10.

3. Phan K, Jo HE, Xu J, Lau EM. Medical Therapy Versus Balloon Angioplasty for CTEPH: A Systematic Review and Meta-Analysis. Heart Lung Circ. 2018;27(1):89-98.

4. Feinstein JA, Goldhaber SZ, LockJE, Ferndandes SM, Landzberg MJ. Balloon pulmonary angioplasty for treatment of chronic thromboembolic pulmonary hypertension. Circulation. 2001;103(1):10-3.

5. Mizoguchi H, Ogawa A, Munemasa M, Mikouchi H, Ito H, Matsubara $\mathrm{H}$. Refined balloon pulmonary angioplasty for inoperable patients with chronic thromboembolic pulmonary hypertension. Circ Cardiovasc Interv. 2012;5(6):748-55

6. Kawakami T, Ogawa A, Miyaji K, Mizoguchi H, Shimokawahara H, Naito T, et al. Novel Angiographic Classification of Each Vascular Lesion in Chronic

\section{Vinculação acadêmica}

Não há vinculação deste estudo a programas de pós-graduação.

\section{Aprovação ética e consentimento informado}

Este artigo não contém estudos com humanos ou animais realizados por nenhum dos autores.

Thromboembolic Pulmonary Hypertension Based on Selective Angiogram and Results of Balloon Pulmonary Angioplasty. Circ Cardiovasc Interv. 2016;9(10):e003318.

7. Ejiri K, Ogawa A, Fujii S, Ito H, Matsubara H. Vascular Injury Is a Major Cause of Lung Injury After Balloon Pulmonary Angioplasty in Patients With Chronic Thromboembolic Pulmonary Hypertension. Circ Cardiovasc Interv. 2018;11(12):e005884.

8. Ikeda N, Kubota S, Okazaki T, Iijima R, Hara H, Hiroi Y, et al. The predictors of complications in balloon pulmonary angioplasty for chronic thromboembolic pulmonary hypertension. Catheter Cardiovasc Interv. 2019:93(6):E349-E356.

9. Sznajder M, Dzikowska-Diduch O, Kurnicka K, Roik M, Wretowski D, Pruszczyk $\mathrm{P}$, et al. Increased systemic arterial stiffness in patients with chronic thromboembolic pulmonary hypertension. Cardiol J. 2018 Sep 20. Doi.10.5603/CJ.a2018.0109

10. Ogawa A, Satoh T, Fukuda T, Sugimura K, Fukumoto Y, Emoto N, et al Balloon Pulmonary Angioplasty for Chronic Thromboembolic Pulmonary Hypertension: Results of a Multicenter Registry. Circ Cardiovasc Qual Outcomes. 2017;10(11): pii: e004029. 